\title{
Cooperative Navigation and Coverage Identification with Random Gossip and Sensor Fusion
}

André Ribeiro Braga, Carsten Fritsche, Marcelo G. S. Bruno and Fredrik Gustafsson

\author{
Linköping University Post Print
}

\section{Tweet}

N.B.: When citing this work, cite the original article.

Original Publication:

André Ribeiro Braga, Carsten Fritsche, Marcelo G. S. Bruno and Fredrik Gustafsson, Cooperative Navigation and Coverage Identification with Random Gossip and Sensor Fusion, 2016, Proc. IEEE 9th Sensor Array and Multichannel Signal Processing Workshop (SAM), 15.

Copyright: http://www.ieee.org

Postprint available at: Linköping University Electronic Press

http://urn.kb.se/resolve?urn=urn:nbn:se:liu:diva-130903 


\section{COOPERATIVE NAVIGATION AND COVERAGE IDENTIFICATION WITH RANDOM GOSSIP AND SENSOR FUSION}

André R. Braga and Marcelo G.S. Bruno

Division of Electronics Engineering

Aeronautics Institute of Technology

São José dos Campos SP 12228-900, Brazil

\author{
Carsten Fritsche and Fredrik Gustafsson
}

\author{
Department of Electrical Engineering \\ Linköping University \\ SE-581 83 Linköping, Sweden
}

\begin{abstract}
This paper is concerned with cooperative Terrain Aided Navigation of a network of aircraft using fusion of Radar Altimeter and inter-node range measurements. State inference is performed using a Rao-Blackwellized Particle Filter with online measurement noise statistics estimation. For terrain coverage measurement noise parameter identification, an online Expectation Maximization algorithm is proposed, where local sufficient statistics at each node are calculated in the E-step, which are then distributed to neighboring nodes using a random gossip algorithm to perform the M-step at each node. Simulation results show that improvement on positioning and calibration performance can be achieved compared to a non-cooperative approach.
\end{abstract}

\section{INTRODUCTION}

Modern autonomous systems are deployed in various environments, such as underwater [1], indoors [2], or in civil and military aviation [3]. Recently, there has been an increased interest in multi-vehicle missions, where each platform acts autonomously to fulfill a specified task. Apart from these tasks, each platform performs self-localization to navigate through the unknown environment, which is usually carried out independently of other platforms. This approach, however, has several shortcomings. For instance, the same external landmarks may be used by different platforms for self-localization, or inter-platform observations are available. Exploiting this type of information generally improves the self-localization of each platform, and therefore navigation of multiple platforms need to be treated as a whole [2], which is called cooperative navigation.

In cooperative navigation, each platform (or node of a global network) has direct access to the observations of its own sensors. Depending on the capacity of the (wireless) communication link, the information collected at each node is

This work was supported by the Vinnova Industry Excellence Center LINK-SIC at Linköping University. André R. Braga was supported by $\mathrm{CNPq}$ - Conselho Nacional de Desenvolvimento Científico e Tecnológico, CISB Centro de Pesquisa e Inovação Sueco-Brasileiro and Saab AB. usually distributed over the network, and each node then locally performs an improved estimation based on the information received from the neighboring nodes. This so-called distributed estimation approach generally leads to an improved global network estimation performance [4]. Consensus algorithms are promising distributed approaches where all nodes in the network aim at reaching an agreement on some common unknown information. The consensus averaging technique [5] has been studied in linear distributed estimation problems using distributed Kalman Filter (KF) [6] and also in nonlinear problems using Particle Filter (PF) [4]. It has the property of asymptotically reaching the solution of the centralized approach, but one of its drawbacks is the potentially prohibitive communication overhead due to the multiiterative consensus step [7,8]. Due to this shortcoming, other techniques have been suggested in the literature which require less communication bandwidth [9].

The purpose of this work is to study the problem of Terrain Aided Navigation (TAN), whose concept is to use terrain height variations along the aircraft flight path to provide high performance position estimates in an autonomous manner without any support information sent to the aircraft $[10,11]$. The ground clearance (or terrain elevation) is measured by the Radar Altimeter (RALT), which is then compared to a terrain height profile map to infer the aircraft's position. The challenge with TAN is that the observation model composed of the terrain height profile map is highly nonlinear and non-analytical, i.e. common filtering approaches such as Extended Kalman Filter (EKF) and Unscented Kalman Filter (UKF) break-down [12]. In addition to that, when measuring the ground clearance, the RALT will sometimes react on echoes from tree tops or buildings, which can be modeled as Gaussian Mixture Model (GMM) noise error whose parameters are generally unknown $[11,12]$.

This paper can be seen as an extension of our previous work [13], where the RALT observation has been modeled as a jump Markov nonlinear system, in order to account for the time-varying nature of the noise statistics. A RaoBlackwellized Particle Filter (RBPF) was then used for each aircraft to simultaneously estimate the aircraft's state together 
with the model parameters describing the RALT observation noise. Cooperation between aircraft took place by exchanging sufficient statistics of the noise model parameters (which can be assumed the same for all aircraft) via a consensus algorithm. The main contributions of this work are as follows: We extend the cooperation among aircraft by additionally considering inter-node range measurements. These provide geometric constraints on the position estimation [1] and increase the reliability of the navigation in case (flat) areas with low information on the terrain elevation profile are overflown. The sufficient statistics of all measurement noise model parameters are distributed based on random pairwise gossip [9].

\section{SYSTEM MODEL}

1) General: The topology of the sensor network is modeled as $\mathcal{G}=(\nu, \varepsilon)$, which is an undirected graphical model where $\nu$ is the set of $N_{S}$ sensor nodes and $\varepsilon$ are the set of edges, each as an unordered pair of distinct nodes. The neighborhood of a node $s \in \nu$ is defined as $\Gamma(s) \triangleq\{r \mid(s, r) \in \varepsilon\}$. Each node $s$ is assumed to be represented by a Jump Markov NonLinear System (JMNLS) of the form

$$
\begin{aligned}
& x_{s, t} \sim f\left(x_{s, t} \mid x_{s, t-1}\right), \\
& y_{s, t} \sim g_{r_{s, t}}\left(y_{s, t} \mid x_{s, t} ; \theta_{r_{s, t}}\right), \\
& r_{s, t} \sim \Pi\left(r_{s, t} \mid r_{s, t-1}\right) .
\end{aligned}
$$

where $x_{s, t} \in \mathbb{R}^{n_{x}}$ denotes the state of node $s$ at time instance $t$ with transition density $f\left(x_{s, t} \mid x_{s, t-1}\right)$, and $r_{s, t} \in$ $\{1, \ldots, K\}$ is a discrete mode variable that evolves according to a Markov chain with $K \times K$ Transition Probability Matrix (TPM), whose elements are assumed to be unknown and given by $\pi_{k \ell}=\Pi(\ell \mid k)=\mathbb{P}\left(r_{t}=\ell \mid r_{t-1}=k\right)$. The state $x_{s, t}$ and the mode $r_{s, t}$ are latent, and are indirectly observed through the measurement $y_{s, t} \in \mathbb{R}^{n_{y}}$ which is defined by a mode-dependent likelihood $g_{r_{s, t}}\left(y_{s, t} \mid x_{s, t} ; \theta_{r_{s, t}}\right)$, with unknown model parameters $\theta_{k}, k=1, \ldots, K$. The goal is then to sequentially estimate $\left(x_{s, t}, r_{s, t}\right)$ and identify the model parameters $\theta=\left(\left\{\theta_{k}\right\}_{k=1}^{K}, \Pi\right)$ from the measurements $y_{s, 0: t}=\left[y_{s, 0}, \ldots, y_{s, t}\right]^{T}$ available up to time $t$.

2) Terrain Based Navigation: We consider $N_{S}$ aircraft in formation flight, representing the sensor network. The dynamics of each aircraft (or node) $s$ given by (1a) are assumed to follow a constant velocity model [14], with state vector $x_{s, t}$ composed of the pairs $\left\{p_{s, t}^{X}, p_{s, t}^{Y}\right\}$ and $\left\{v_{s, t}^{X}, v_{s, t}^{Y}\right\}$ that represent the $2 \mathrm{D}$ position and velocity. Each aircraft is measuring the height above the terrain from the RALT (altitude above sea level known), modeled as $y_{s, t}=h_{1}\left(p_{s, t}^{X}, p_{s, t}^{Y}\right)+e_{r_{s, t}}$, where $h_{1}(.,$.$) is a non-analytical and nonlinear lookup ta-$ ble that represents the terrain elevation database that is stored in the aircraft's computer. The observation noise $e_{r_{s, t}}$ is assumed mode-dependent, to account for the effect of multiple reflections of the RALT echo signal on the open terrain model [15]. The echo reflections are modeled by a 2-state Markov chain [16], that switches between two Gaussian distributions each having unknown mean and standard deviation, i.e. $\theta_{k}=$ $\left\{\mu_{k}, \sigma_{k}\right\}, k=1,2$. In addition to the RALT observations, each node takes inter-node range measurements (via the internode communication data link) to its neighboring sensors, which are modeled as $\tilde{y}_{s r, t}=h_{2}\left(p_{s, t}^{X}, p_{s, t}^{Y}, p_{r, t}^{X}, p_{r, t}^{Y}\right)+\tilde{e}_{s, t}$ with $r \in \Gamma(s)$ and where $h_{2}(., ., .,$.$) denotes the Euclidean$ distance between $\left\{p_{s, t}^{X}, p_{s, t}^{Y}\right\}$ and $\left\{p_{r, t}^{X}, p_{r, t}^{Y}\right\}$. We further assume reciprocity of the communication channel, i.e. $\tilde{y}_{s r, t}=$ $\tilde{y}_{r s, t}$ holds, and the noise $\tilde{e}_{s, t}$ can be assumed zero-mean Gaussian distributed with known variance $\sigma_{d}^{2}$. Note, that the variance can be also treated as an unknown parameter that has to be identified, but we refrain from this option in the following.

\section{PROPOSED SOLUTION}

1) Cooperative Sequential State Estimation: We assume that at every time instant $t$ each node $s$ receives the following observation vector $Y_{s, t} \triangleq\left[\begin{array}{lll}y_{s, t} & \bar{Y}_{s, t} & \widetilde{Y}_{s, t}\end{array}\right]^{T}$, where $y_{s, t}$ is terrain elevation measurement observed by node $s$, $\bar{Y}_{s, t} \triangleq\left\{y_{r, t}\right\}_{r \in \Gamma(s)}$ are the terrain elevation measurements of neighboring nodes, and $\tilde{Y}_{s, t} \triangleq\left\{\tilde{y}_{s r, t}\right\}_{r \in \Gamma(s)}$ is the set of inter-node range measurements between node $s$ and its neighbors. For notational simplicity, we drop the unknown parameter $\theta$ from the notation throughout this section. From a Bayesian perspective, we are interested in recursively evaluating

$$
p\left(x_{s, 1: t}, r_{s, t} \mid Y_{s, 1: t}\right)=\mathbb{P}\left(r_{s, t} \mid x_{s, 1: t}, Y_{s, 1: t}\right) p\left(x_{s, 1: t} \mid Y_{s, 1: t}\right)
$$

where the first density can be evaluated analytically using conditional Hidden Markov Model (HMM) filters, and the density $p\left(x_{s, 1: t} \mid Y_{s, 1: t}\right)$ can be approximated using particle filters [17]. This technique is known as Rao-Blackwellization and generally can lead to a reduction in variance of the estimated parameters. The derivation of the filter can be found in $[13,17]$ and is not repeated here. Rather, we present in the following only the required filter modifications. We define for each particle $i$ (represented by the superscript $(i)$ ) the quantity

$$
\begin{aligned}
\gamma_{s, t}^{(i)}\left(r_{s, t}\right) & \triangleq p\left(Y_{s, t}, x_{s, t}^{(i)}, r_{s, t} \mid x_{s, 1: t-1}^{(i)}, Y_{s, 1: t-1}\right) \\
& =p_{r_{s, t}}\left(Y_{s, t} \mid x_{s, t}^{(i)}\right) f\left(x_{s, t}^{(i)} \mid x_{s, t-1}^{(i)}\right) \alpha_{s, t \mid t-1}^{(i)}\left(r_{s, t}\right) .
\end{aligned}
$$

where $\alpha_{s, t \mid t-1}^{(i)}(\ell) \triangleq \mathbb{P}\left(r_{s, t}=\ell \mid x_{s, 1: t-1}^{(i)}, Y_{s, 1: t-1}\right)$ is obtained by prediction using the previous HMM filter output $\alpha_{s, t-1 \mid t-1}^{(i)}(\ell)$ for $\ell \in\{1, \ldots, K\}$. The HMM filter update is then given by $\alpha_{s, t \mid t}^{(i)}(\ell)=\gamma_{s, t}^{(i)}(\ell) / \sum_{k=1}^{K} \gamma_{s, t}^{(i)}(k)$. The unnormalised particle weights $\tilde{w}_{s, t}^{(i)}$ can be adjusted accordingly, 
yielding

$$
\tilde{w}_{s, t}^{(i)} \propto w_{s, t-1}^{(i)} \frac{p\left(x_{s, t}^{(i)}, Y_{s, t} \mid x_{s, 1: t-1}^{(i)}, Y_{s, 1: t-1}\right)}{q\left(x_{s, t}^{(i)} \mid x_{s, 1: t-1}^{(i)}, Y_{s, 1: t-1}\right)}
$$

with $p\left(x_{s, t}^{(i)}, Y_{s, t} \mid x_{s, 1: t-1}^{(i)}, Y_{s, 1: t-1}\right)=\sum_{k=1}^{K} \gamma_{s, t}^{(i)}(k)$, and where $q\left(x_{s, t}^{(i)} \mid x_{s, 1: t-1}^{(i)}, Y_{s, 1: t-1}\right)$ denotes the proposal density.

The modified likelihood $p_{r_{s, t}}\left(Y_{s, t} \mid x_{s, t}\right)$ can be decomposed as follows

$$
\begin{aligned}
p_{r_{s, t}}\left(Y_{s, t} \mid x_{s, t}\right) & =g_{r_{s, t}}\left(y_{s, t} \mid x_{s, t}\right) p\left(\bar{Y}_{s, t}, \tilde{Y}_{s, t} \mid x_{s, t}\right) \\
& =g_{r_{s, t}}\left(y_{s, t} \mid x_{s, t}\right) \prod_{r \in \Gamma(s)} p\left(y_{r, t}, \tilde{y}_{s r, t} \mid x_{s, t}\right)
\end{aligned}
$$

where the second equality follows from the assumption that the observations are mutually independent. We further rewrite the density $p\left(y_{r, t}, \tilde{y}_{s r, t} \mid x_{s, t}\right)$ in terms of the state vector $x_{r, t}$ of the neighboring node

$$
\begin{aligned}
& p\left(y_{r, t}, \tilde{y}_{s r, t} \mid x_{s, t}\right)=\int p\left(y_{r, t}, \tilde{y}_{s r, t}, x_{r, t} \mid x_{s, t}\right) \mathrm{d} x_{r, t} \\
& =\int p\left(y_{r, t} \mid x_{r, t}\right) p\left(\tilde{y}_{s r, t} \mid x_{r, t}, x_{s, t}\right) p\left(x_{r, t} \mid x_{s, t}\right) \mathrm{d} x_{r, t} .
\end{aligned}
$$

The expression above is generally difficult to evaluate, so that further simplifying assumptions have to be introduced. In particular, the evaluation of $p\left(y_{r, t} \mid x_{r, t}\right)$ requires knowledge of the mode $r_{r, t}$ which has generated the observation. In the following we approximate this density by a mixture

$$
p\left(y_{r, t} \mid x_{r, t}\right) \approx \frac{1}{K} \sum_{k=1}^{K} g_{k}\left(y_{r, t} \mid x_{r, t}\right)
$$

where we have assumed equal probable mixture component weights. It is also possible to replace the mixture component weights with conditional mode estimates $\alpha_{r, t \mid t}(k)$ available from the RBPF of node $r$, but this requires further information exchange via the communication channel. Another issue is the assumption on the density $p\left(x_{r, t} \mid x_{s, t}\right)$. It is possible to be considered as uniform distributed over some space relative to node $s$, since we do not have (and exchange) information about the current state of node $r$. Instead of evaluating the integral (6) over the entire space, we follow an approach that has been suggested in [18]. We first note that the models for $y_{r, t}$ and $\tilde{y}_{s r, t}$ are independent of the $2 \mathrm{D}$ velocity. Hence, the integration in (6) can be performed over the $2 \mathrm{D}$ position space. We consider a smaller, ring-shaped discrete grid, $\chi_{r}$, which is centered at the position of node $s$ and which has a radius equal to the measured inter-node range $\tilde{y}_{s r, t}$. The width of the ring is based on a confidence interval of the inter-node range observation model and is assumed to be $\pm 3 \sigma_{d}^{2}$. Assuming $N_{G}$ grid points on the ring, the density can be approximated numerically according to

$$
p\left(y_{r, t}, \tilde{y}_{s r, t} \mid x_{s, t}\right) \approx \frac{1}{N_{G}} \sum_{x_{r, t}^{(j)} \in \chi_{r}} p\left(\tilde{y}_{s r, t} \mid x_{r, t}^{(j)}, x_{s, t}\right) p\left(y_{r, t} \mid x_{r, t}^{(j)}\right) .
$$

2) Networked Parameter Estimation: For estimating the unknown parameter vector $\theta$, the online Expectation Maximization (EM) algorithm [19] is used. It requires that the nonlinear dynamical system corresponding to each mode belongs to the curved exponential family [20], such that a recursive calculation of the corresponding sufficient statistics $s\left(z_{s, t}, z_{s, t-1}\right)$, with $z_{s, t} \triangleq\left\{x_{s, t}, r_{s, t}\right\}$, becomes feasible. In particular, it enables to recursively update an intermediate quantity $T_{s, t}\left(z_{s, t}\right) \triangleq \mathbb{E}_{\theta^{\prime}}\left[\sum_{t=1}^{n} s\left(z_{s, t}, z_{s, t-1}\right) \mid z_{s, t}, y_{s, 1: t}\right]$ at each time step, from which the a new parameter estimate $\hat{\theta}^{t}$ can be computed from the mapping $\Lambda_{k}($.$) , as it is described in$ more detail in [13,21]. The RBPF output, i.e. $\left\{w_{s, t}^{(i)}, x_{s, t}^{(i)}\right\}_{i=1}^{N_{p}}$ can be used to compute for each particle an approximation of the intermediate quantity $\widehat{T}_{s, t}^{(i)}(\ell) \approx T_{s, t}\left(x_{s, t}^{(i)}, r_{s, t}=\ell\right)$, according to [17]

$$
\widehat{T}_{s, t}^{(i)}(\ell)=\sum_{j=1}^{N_{P}} \sum_{k=1}^{K}\left(\frac{\widetilde{w}_{s, t}^{(i, j)}(\ell, k)}{\sum_{u=1}^{N} \sum_{m=1}^{K} \widetilde{w}_{s, t}^{(i, u)}(\ell, m)}\right.
$$

$\left.\times\left[\left(1-\eta_{t}\right) \widehat{T}_{s, t-1}^{(j)}(k)+\eta_{t} s_{t}\left(x_{s, t-1}^{(j)}, r_{s, t-1}=k, x_{s, t}^{(i)}, r_{s, t}=\ell\right)\right]\right)$

where

$$
\widetilde{w}_{s, t}^{(i, j)}(\ell, k)=f\left(x_{s, t}^{(i)} \mid x_{s, t-1}^{(j)}\right) \pi_{k \ell} \alpha_{s, t-1 \mid t-1}^{(j)}(k) w_{s, t-1}^{(j)},
$$

the recursion is initialized with $\widehat{T}_{s, 0}^{(i)}(\ell)=0$, and where $\eta_{t}$ is a forgetting factor satisfying the stochastic approximation requirements $\sum_{t \geq 1} \eta_{t}=\infty$ and $\sum_{t \geq 1} \eta_{t}^{2}<\infty$. The estimate for the local sufficient statistics is finally obtained from $\hat{\mathcal{S}}_{s, t}=$ $\sum_{i=1}^{N_{P}} \sum_{\ell=1}^{K} w_{s, t}^{(i)} \alpha_{s, t \mid t}^{(i)} \hat{T}_{s, t}^{(i)}(\ell)$. The computational complexity of computing (9) is $\mathcal{O}\left(K^{2} N_{P}^{2}\right)$ and can be reduced by using path-based smoothing [17], which is however not considered in this work.

2.1) Random Gossip: Defined as an algorithm in which each node communicates with no more than one neighbor in each time slot [9]. Different from consensus, evaluated in our previous work [13], when each node $s$ communicates with all the elements of $\Gamma(s)$, this algorithm utilizes only a subset of unitary size.

As the quantity of interest we consider the sufficient statistics, whose distributed averaging is aimed. Within this approach, at each time-step, a randomly selected set of pairs from neighboring nodes exchange data in order to spread information throughout the network. The information exchanged between node pairs (local sufficient statistics) is 
combined as $\zeta_{s}^{(t)}=\frac{1}{2}\left(\hat{\mathcal{S}}_{s, t}+\hat{\mathcal{S}}_{r, t}\right)$ and leads to a random information spread over the network.

It is worth noting that the random gossip is applied only to the parameter estimation. Cooperation via inter-node measurments is performed over standard protocol where each node is communicating with each other.

The random gossip approach can significantly reduce the communication cost, since, instead of communicating iteratively with all the neighbours, each node has only to exchange information with one single node.

\section{SIMULATIONS}

Performance is assessed via 100 independent Monte Carlo runs evaluating a 22.5 seconds (450 measurements) of stabilized flight from $N_{S}=9$ aircraft flying in constant formation over a simulated terrain created by a 2D Gaussian low-pass filter over uniform noise [22]. The mode-dependent observation noise of the RALT follows a 2-state Markov chain, with transition probabilities $\pi_{11}=0.85$ and $\pi_{22}=0.6$, that switches between two Gaussian distributions with parameters $\theta_{1}=\{0,1\}$ and $\theta_{2}=\{20,4\}$. For the inter-node measurements, $\sigma_{d}=0.5$. The forgetting factor follows $\eta_{t}=t^{-0.7}$.

The RBPF for each node uses $N_{P}=300$ particles, a blind proposal density and $N_{G}=400$ grid points for the internode measurements likelihood evaluation. At time $t=0$, all filters are initialized with $x_{s, 0}^{(i)}=x_{s, 0}, w_{s, 0}^{(i)}=1 / N_{P}$ and $\alpha_{s, 0\rfloor 0}^{(i)}(\ell)=\mathbb{1}\left(r_{s, 0}=\ell\right)$ for $s=1, \ldots, N_{S}$ and $i=$ $1, \ldots, N_{P}$. The parameter estimation starts after 2.5 seconds (50 iterations) in order to guarantee that the M-step update is numerically well-behaved [21]. The initial guess for the online EM algorithm are given by: $\hat{\pi}_{11}^{0}=0.5$ and $\hat{\pi}_{22}^{0}=0.5$, $\hat{\theta}_{1}^{0}=\{-5,5\}$ and $\hat{\theta}_{2}^{0}=\{25,5\}$.

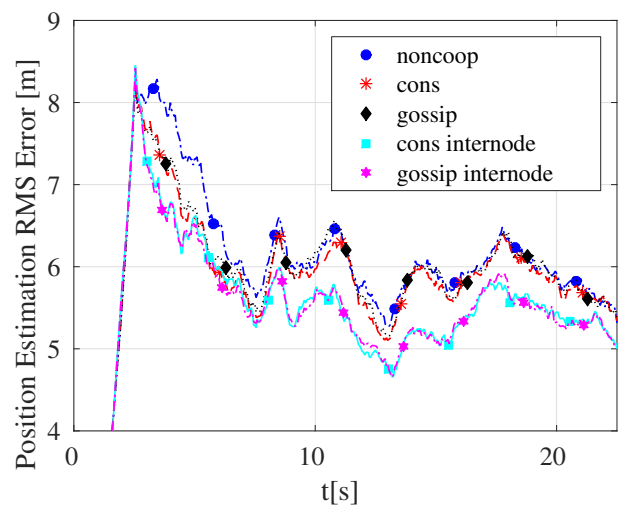

Fig. 1. Position RMS error.

1) Results Analysis: Both consensus and random gossip strategies were evaluated considering only one iteration per time step. It is then the minimum load on the network imposed by the parameter estimation task. Figure 1 addresses the position estimation Root Mean Square (RMS) error. It is clear the proposed solution for the cooperative filtering provides some performance increase on the navigation task (around 12\%).

In Figure 2, the parameter estimation presents substantial performance improvement when using cooperation between nodes. The random gossip behaves almost as the consensus strategy.
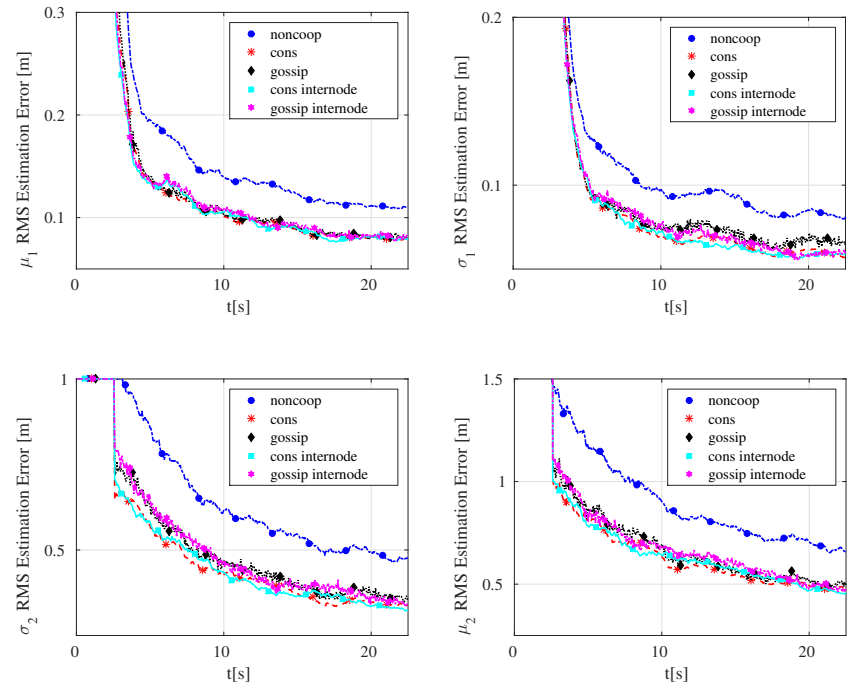

Fig. 2. Parameters RMS estimation error.

2) Communication Analysis: The total communication bandwidth required by the entire network, also called network throughput, was analysed for each strategy. We assume a four-Byte representation for a floating point value and the set of sufficient statistics demand 10 values (40 Bytes). The consensus approach demands $18.75 \mathrm{~KB} / \mathrm{s}$ and the random gossip $6.25 \mathrm{~KB} / \mathrm{s}$ in network throughput. A reduction of more than $60 \%$ for parameter estimation can be achieved.

Regarding the use of inter-node measurements, the demand increases to $20.625 \mathrm{~KB} / \mathrm{s}$ and $7.1875 \mathrm{~KB} / \mathrm{s}$ for the consensus and random gossip, respectively, due to the exchange of an additional floating point value between neighbors $(10 \%$ and $15 \%$ bandwidth increase, respectively). We have then a trade off between navigation accuracy and network communication resources usage.

\section{CONCLUSION}

We have proposed two cooperative approaches for both filtering and model parameters estimation. The results on a network of flying platforms show that cooperation using the randomized gossip over the sufficient statistics exchange provides similar performance compared to the consensus approach using significant less network bandwidth. The state estimation takes advantage of the inter-node measurements together with the exchange of RALT measurements. 


\section{REFERENCES}

[1] T. Y. Teck, M. Chitre, and F. S. Hover, "Collaborative Bathymetry-Based Localization of a Team of Autonomous Underwater Vehicles," in Robotics and Automation (ICRA), 2014 IEEE International Conference on, May 2014, pp. 2475-2481.

[2] H. Mu, T. Bailey, P. Thompson, and H. Durrant-Whyte, "Decentralised Solutions to the Cooperative MultiPlatform Navigation Problem," Aerospace and Electronic Systems, IEEE Transactions on, vol. 47, no. 2, pp. 1433-1449, April 2011.

[3] D. Magnusson, "A Network Based Algorithm for Aided Navigation,” Tech. Rep. Master's Thesis No LiTH-ISYEX-11/4534-SE, 2012.

[4] O. Hlinka, F. Hlawatsch, and P. Djuric, "Distributed Particle Filtering in Agent Networks: A Survey, Classification, and Comparison," Signal Processing Magazine, IEEE, vol. 30, no. 1, pp. 61-81, Jan 2013.

[5] L. Xiao and S. Boyd, "Fast Linear Iterations for Distributed Averaging," Systems and Control Letters, vol. 53, pp. 65-78, 2003.

[6] I. Schizas, G. Giannakis, S. Roumeliotis, and A. Ribeiro, "Consensus in Ad Hoc WSNs With Noisy Links - Part II: Distributed Estimation and Smoothing of Random Signals," Signal Processing, IEEE Transactions on, vol. 56, no. 4, pp. 1650-1666, April 2008.

[7] K. Dedecius, J. Reichl, and P. Djuric, "Sequential Estimation of Mixtures in Diffusion Networks," Signal Processing Letters, IEEE, vol. 22, no. 2, pp. 197-201, Feb 2015.

[8] S. Y. Tu and A. H. Sayed, "Diffusion Strategies Outperform Consensus Strategies for Distributed Estimation Over Adaptive Networks," IEEE Transactions on Signal Processing, vol. 60, no. 12, pp. 6217-6234, Dec 2012.

[9] S. Boyd, A. Ghosh, B. Prabhakar, and D. Shah, "Randomized Gossip Algorithms," IEEE Transactions on Information Theory, vol. 52, no. 6, pp. 2508-2530, June 2006.

[10] A. Henley, “Terrain Aided Navigation: Current Status, Techniques for Flat Terrain and Reference Data Requirements," in Position Location and Navigation Symposium, 1990. Record. The 1990's - A Decade of Excellence in the Navigation Sciences. IEEE PLANS '90., IEEE, Mar 1990, pp. 608-615.

[11] N. Bergman, L. Ljung, and F. Gustafsson, "Terrain Navigation Using Bayesian Statistics," Control Systems, IEEE, vol. 19, no. 3, pp. 33-40, Jun 1999.
[12] P.-J. Nordlund and F. Gustafsson, "Marginalized Particle Filter for Accurate and Reliable Terrain-Aided Navigation," Aerospace and Electronic Systems, IEEE Transactions on, vol. 45, no. 4, pp. 1385-1399, Oct 2009.

[13] A. R. Braga, E. Özkan, C. Fritsche, F. Gustafsson, and M. Bruno, "Cooperative Terrain Based Navigation and Coverage Identification Using Consensus," in 18th International Conference on Information Fusion (Fusion 2015), Washington, USA, Jul. 2015.

[14] X. Li and V. Jilkov, "Survey of Maneuvering Target Tracking. Part I. Dynamic Models," Aerospace and Electronic Systems, IEEE Transactions on, vol. 39, no. 4, pp. 1333-1364, Oct 2003.

[15] C. Dahlgren, Nonlinear Black Box Modelling of JAS 39 Gripen's Radar Altimeter, ser. LiTH-ISY-Ex: 1958. Linköpings Universited, 1998.

[16] P. Frykman, Applied Particle Filters in Integrated Aircraft Navigation., ser. LiTH-ISY-Ex: 3406. Linköpings Universited, 2003.

[17] E. Özkan, F. Lindsten, C. Fritsche, and F. Gustafsson, "Recursive Maximum Likelihood Identification of Jump Markov Nonlinear Systems," Signal Processing, IEEE Transactions on, vol. 63, no. 3, pp. 754-765, Feb 2015.

[18] L. Baglivo, “Cooperative Terrain-Aided Navigation AUVs using Particle Filters," Universidade de Aveiro, Tech. Rep. CESAM-DGEO-CTAN-13-01, 2013.

[19] O. Cappe, “Online Sequential Monte Carlo EM Algorithm," in Statistical Signal Processing, 2009. SSP '09. IEEE/SP 15th Workshop on, Aug 2009, pp. 37-40.

[20] P. Del Moral, A. Doucet, and S. Singh, "Forward Smoothing using Sequential Monte Carlo," Cambridge University Engineering Department, Tech. Rep. CUED/F-INFENG/TR638, 2010.

[21] O. Cappe, "Online EM Algorithm for Hidden Markov Models," Journal of Computational and Graphical Statistics, vol. 20, no. 3, pp. 728-749, 2011.

[22] F. Gustafsson, "Particle Filter Theory and Practice with Positioning Applications," Aerospace and Electronic Systems Magazine, IEEE, vol. 25, no. 7, pp. 53-82, July 2010. 